\title{
An Application-Oriented Network Model for Wireless Sensor Networks*
}

\author{
Xiaoliang Cheng, Zhidong Deng ${ }^{\#}$, Zhen Huang \\ State Key Laboratory of Intelligent Technology and Systems, Tsinghua National Laboratory for Information Science \\ and Technology, Department of Computer Science, Tsinghua University, Beijing, China \\ E-mail: chengxl06@mails.tsinghua.edu.cn,michael@mail.tsinghua.edu.cn, chinaheart2003@qq.com \\ Received June 29, 2010; revised August 2, 2010; accepted September 10, 2010
}

\begin{abstract}
Wireless sensor networks (WSNs) are energy-constrained networks. The residual energy real-time monitoring (RERM) is very important for WSNs. Moreover, network model is an important foundation of RERM research at personal area network (PAN) level. Because RERM is inherently application-oriented, the network model adopted should also be application-oriented. However, many factors of WSNs applications such as link selected probability and ACK mechanism etc. were neglected by current network models. These factors can introduce obvious influence on throughput of WSNs. Then the energy consumption of nodes will be influenced greatly. So these models cannot characterize many real properties of WSNs, and the result of RERM is not consistent with the real-world situation. In this study, these factors neglected by other researchers are taken into account. Furthermore, an application-oriented general network model (AGNM) for RERM is proposed. Based on the AGNM, the dynamic characteristics of WSNs are simulated. The experimental results show that AGNM can approximately characterize the real situation of WSNs. Therefore, the AGNM provides a good foundation for RERM research.
\end{abstract}

Keywords: Wireless Sensor Networks, Personal Area Network (PAN), Network Model, Throughput Analysis

\section{Introduction}

With the rapid development of WSNs [1-3], the interaction manners between the human being world and the physical world have been changed greatly. Utilizing welldeployed WSNs, we can sense the selected physical world at anytime; then we could exert some customized influences on the physical world. Therefore, WSNs are attractting increasing interests of people.

However, WSNs are resource-constrained networks. These constraints are embodied almost at every aspect of WSNs [4]. The large-scale deployment of WSNs is constrained by these factors, especially in terms of energy. To monitor the energy consumption of WSNs, the RERM research becomes an urgent task. Moreover, network model is the fundamental infrastructure of RERM research at the PAN-level. Because the RERM research is inherently application-oriented, the network model adopted should be application-oriented too.

*Supported by the National High Technology Development 863 Program of China under Grant No. 2006AA040102.
There are lots of factors that can influence the energy consumption of WSNs. These factors have a large transitional range in link quality that is characterized by significant levels of unreliability and asymmetry [5]. Moreover, these characteristics have also been verified in our previous study [6]. However, these properties of WSNs have not been handled by the network models adopted by other previous RERM researches reported [7-10]. This becomes the main reasons of the inconsistent between RERM results and the real WSNs.

To solve this problem, based on real platforms, a new perspective of PAN was investigated by us when analyzing the fundamental causes of unreliability and asymmetry. This model takes into account factors such as link selected probability and ACK mechanism, etc. Then, an application-oriented general network model (AGNM) used for RERM research was proposed. The simulative results showed that AGNM can approximately characterize the real situation of WSNs.

The rest of this paper is organized as follows. A brief introduction of the research platform is introduced in 
Section 2. AGNM is proposed in Section 3. In Section 4, some simulation experiments are carried out to testify the effectiveness of AGNM. Section 5 concludes this paper and suggests some future research topics.

\section{Research Platform}

The RERM research should be application-oriented. To effectively study RERM, an application-oriented general network model for WSNs (AGNM) should be proposed first. The AGNM model should be established based on appropriate platform and real application environment. To our best knowledge, the ZigBee/802.15.4 specification reflects the basic characteristics of WSNs better than other specifications $[4,11]$. The BeeStack protocol stack is complied with ZigBee/802.15.4 [12]. So, the ZigBee/ 802.15.4 and BeeStack protocols are selected as our research platforms.

ZigBee [13] is based on 802.15.4 [14]. The medium access control (MAC) layer and physical layer (PHY) were defined by 802.15.4; and the upper layers from network layer (NWK) were defined by ZigBee. Especially, the NWK layer of ZigBee provides full support for Star, Cluster-Tree and Mesh topologies.

In the ZigBee specification, nodes are categorized into three types: coordinator $(\mathrm{ZC})$, router $(\mathrm{ZR})$ and end device (ZED). ZC creates and maintains a PAN. ZRs route data to $Z C$ and help maintaining a PAN. ZEDs sense the physical world and send information to ZC with the help of ZRs. Moreover, each node maintains a neighbor table locally. After the node has joined a PAN, its neighbor table will be used to store relationship and link-state information about neighbor nodes. The table entry of a node should be updated when any frame is received from its neighbors.

Based on this platform, some assumptions can be drawn out. Then, an application-oriented network model can be established.

\section{Application-Oriented General Network Model}

If all the nodes have good consistency, then the energy consumption of each node is mainly determined by its throughputs. Moreover, the throughput is influenced by many other factors. From a perspective of a PAN, these factors can be categorized into two classes: PAN-outside factors (electromagnetic environment etc.), PAN-inside factors (topology, ACK mechanism etc.).

For simplicity, only the PAN-inside factors are discussed here. The PAN-inside factors can be subdivided into two subclasses: the topology factors and the operational factors. Both of them are dynamic and uncertain, especially in the case of Mesh topology.

\subsection{Modeling of Topology Factors}

All the monitoring frameworks proposed in [7-10] cannot provide any support for Mesh topology. Moreover, they cannot be used for the mobile nodes. Hence, their scalability decreased greatly. To solve these problems, this subsection will propose a dynamic topology model based the analysis of ZigBee Mesh topology.

1) Analysis of ZigBee Mesh Topology: A typical ZigBee Mesh topology was introduced as Figure 1.

In Figure 1, dark circle denotes $\mathrm{ZC}$ node; gray circles denote ZR nodes; white circles denote ZED nodes. Lines denote bidirectional radio links. Especially, links among node-1, 2, 3 and 4 form a typical mesh structure. Note that a path loop among nodes should be forbidden, i.e., a frame should not pass through the same node more than once in a delivery path. Let the length of path from node $i$ to $\mathrm{ZC}$ be $L_{i}$, and the minimum hops from node $i$ to $\mathrm{ZC}$ be $D_{i}$, then we adopt the following constraint: $D_{i} \leq L_{i} \leq D_{i}+1$.

In Figure 2, the paths of query-message are depicted by downward arrow lines that start from node-1 (ZC). The relationships among neighbor nodes are created during topology formation. In ZigBee, there are three relationship types: parent, child and sibling. Here, a parent emits a unidirectional arrow line; a child is injected by a unidirectional arrow line; siblings are connected by bidirectional arrow line, because sibling is a mutual relationship type. For convenience, some additional relationship types are defined by us.

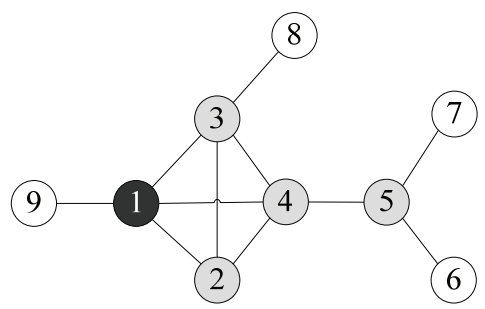

Figure 1. A typical ZigBee Mesh topology.

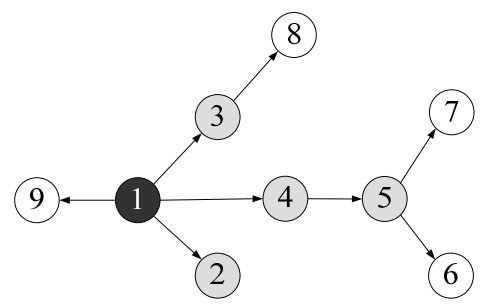

Figure 2. Topology-construction and message-query in ZigBee Mesh topology (downlinks). 
Definition 1: Given the node $i$, all its children (denoted as $\operatorname{ch}(i))$ and the descendants of its children are named as descendants of node $i$ (denoted as $d e(i)$ ), then

$$
\operatorname{de}(i)=\{\operatorname{ch}(i), \operatorname{de}(\operatorname{ch}(i))\} \quad(i \in[1, n])
$$

Definition 2: Given the node $i$, the neighbors of node $i$ (denoted as ne(i) ) comprise all the nodes that can communicate with node $i$ within one hop distance, then

$$
n e(i)=\{p a(i), s i(i), \operatorname{ch}(i)\} \quad(i \in[1, n])
$$

where $p a(i)$ denotes the parents of node $i ; s i(i)$ denotes the siblings of node $i$.

In Figure 3, the paths of report-messages are depicted by upward arrow lines that start from ZEDs/ ZRs and direct to node-1 (destination). For example, when a message is sent from node- 4 to node- 1 , there are five path options: $4 \rightarrow 1, \quad 4 \rightarrow 2 \rightarrow 1, \quad 4 \rightarrow 3 \rightarrow 1$, $4 \rightarrow 2 \rightarrow 3 \rightarrow 1$ and $4 \rightarrow 3 \rightarrow 2 \rightarrow 1$.

According to $D_{i} \leq L_{i} \leq D_{i}+1$, the optional paths are reduced to three ones: $4 \rightarrow 1,4 \rightarrow 2 \rightarrow 1,4 \rightarrow 3 \rightarrow 1$. Different from the case of Cluster-Tree topology, the selection of next hop node in Mesh topology is a probability event

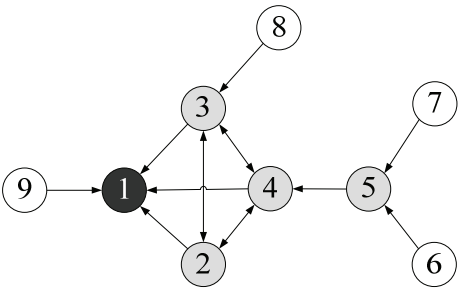

Figure 3. Message-report in ZigBee Mesh topology (uplinks).

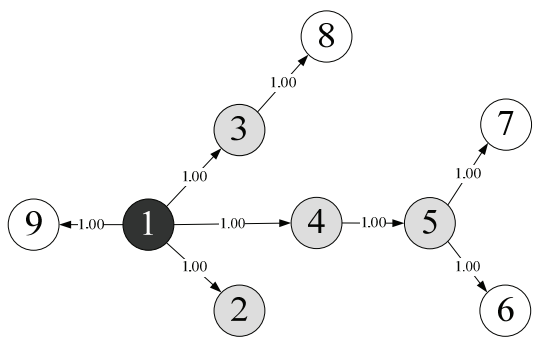

(a)

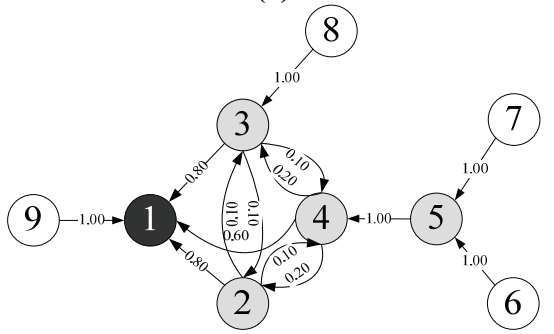

(b)

Figure 4. The link selected probability distribution in a ZigBee Mesh topology. (a) Case of downlinks. (b) Case of uplinks.
[15]. So the state of network topology behaves with evident dynamic characteristics during running time, especially in the case of uplinks. Therefore, the link selected probability is chosen to model the link state (see Figure 4).

The bidirectional link of Figure 1 is subdivided into an uplink and a downlink in Figure 4. Both of them are attached with a selected probability. Specifically, the links $2 \longleftrightarrow 3,2 \longleftrightarrow \longrightarrow 4$ and $3 \longleftrightarrow \longrightarrow 4$ of Figure 4(b) are all uplinks, e.g. $3 \leftarrow \rightarrow 4$ denotes two uplinks: $3 \leftarrow 4$ and $3 \rightarrow 4$.

When a message is sent from node- 4 to node- 1 , the optional paths include: $4 \rightarrow 1,4 \rightarrow 2 \rightarrow 1$ and $4 \rightarrow 3 \rightarrow 1$. The link selected probability distribution of the next hop is $\left\{\mathrm{p}_{4,1}=0.6, \mathrm{p}_{4,2}=0.2\right.$ and $\left.\mathrm{p}_{4,3}=0.2\right\}$. Note that according to $D_{i} \leq L_{i} \leq D_{i}+1$, when node- 1 has been selected as the next hop of node- 4 , the link selected probability distribution should be $\left\{\mathrm{p}_{2,1}=1, \mathrm{p}_{1,3}=0\right.$ and $\left.\mathrm{p}_{2,4}=0\right\}$, instead of $\left\{\mathrm{p}_{2,1}=0.8, \mathrm{p}_{1,3}=0.1\right.$ and $\left.\mathrm{p}_{2,4}=0.1\right\}$.

2) ZigBee Mesh Topology Model: Based on the discussion above, the mathematical models for undirected links, node relationships and link selected probabilities are discussed separately in the following.

a) Undirected Link Model: Based on Graph Theory [16], the undirected link model (see Figure 1) can be defined as an undirected graph $G_{t}=(V, E)$. Here, $V$ is a finite nonempty set, and the elements of $V$ is called vertices; $E$ is a set of two element subsets of $V$, and the elements of $E$ are called edges. In a static topology model, each node corresponds to a vertex; and each link corresponds to an edge. Also, an edge directed from $v_{i}$ to $v_{j}$ is denoted as $<v_{i}, v_{j}>$.

Suppose the number of vertices is $n, G_{t}=(V, E)$ can be expressed as a $n \times n$ adjacency matrix $A_{G-t}$. The elements of $A_{G-t}$ are determined by Formula (3).

$$
a_{i j}= \begin{cases}1 & \text { if }<v_{i}, v_{j}>\in E \\ 0 & \text { if }<v_{i}, v_{j}>\notin E\end{cases}
$$

Note that in an adjacency matrix, the sum of row $i$ is named as out-degree (denoted as $\left.\operatorname{od}\left(v_{i}\right)\right)$, which denotes the number of emitted links from node $i$; the sum of column $i$ is named as in-degree (denoted as $\operatorname{id}\left(v_{i}\right)$ ), which denotes the number of links injected into node $i$. Also, the sum of out-degree and in-degree is named as degree of $i$, i.e. $\operatorname{deg}\left(v_{i}\right)=\operatorname{od}\left(v_{i}\right)+\mathrm{id}\left(v_{i}\right) . A_{G-t}$ is a symmetric matrix, so, $\operatorname{od}\left(v_{i}\right)=\operatorname{id}\left(v_{i}\right)$.

b) Node Relationship Model: The Node Relationship Model (see Figures 2,3) can be defined as a directed graph $G_{r}=(V, E)$. It can be expressed as a $n \times n$ adjacency matrix $A_{G-r}$. The elements of $A_{G-r}$ are determined by Formula (4).

$$
b_{i j}= \begin{cases}1 & \text { if }<v_{i}, v_{j}>\in E \\ 0 & \text { if }<v_{i}, v_{j}>\notin E\end{cases}
$$


When $b_{i j}=1$ and $b_{j i}=0, i$ is a parent of $j$, and $j$ is a child of $i$; when $b_{i j}=b_{j i}=1, i$ and $j$ are siblings. $A_{G-r}$ is an asymmetric matrix.

c) Link Selected Probability Model: Because downlinks (see Figures 2 and 4(a)) take very low portion in the lifetime of WSNs, only the uplink selected probability model is discussed here.

An uplink selected probability model (see Figure 4(b)) can be defined as a selection-weighted directed graph $G(u)_{w e}=(V, E)$. An uplink selected probability as a weight is attached to corresponding edge.

Definition 3: For uplinks, given the node $i$, only the parent and siblings of node $i$ can be selected as the next hop. The set of uplink next hop nodes of node $i$ is denoted as $N u_{i}$, then

$$
N u_{i}=\{p a(i), \operatorname{si}(i)\} \quad(i \in[1, n])
$$

Suppose the selected probability of an uplink from node $i$ to node $j$ is $p(u)_{i j}$, then

$$
p(u)_{i j}=\left\{\begin{array}{cc}
{[0,1]} & \left(j \in N u_{i}\right) \\
0 & \left(j \notin N u_{i}\right)
\end{array}\right.
$$

Moreover,

$$
\sum_{j \in N u_{i}} p(u)_{i j}=1
$$

According to Formulas (6) and (7), $G(u)_{w e}=(V, E)$ (see Figure 4(b)) can be expressed as a $n \times n$ asymmetric adjacency matrix $A_{U-l}$.

\subsection{Modeling of Operational Factors}

The operational factors comprise ACK and retransmission mechanisms, etc. For simplicity, only the influence caused by ACK mechanism is discussed here.

1) Analysis of Throughput: From the perspective of a node, the throughput can be divided into two classes: Throughput of neighbors and throughput of current node. There is a competition between them. The throughput of current node can be further subdivided into in-throughput and out-throughput. Moreover, both in-throughput and out-throughput of current node are influenced by its neighbors.

To guarantee reliable delivery, a fully acknowledged mechanism was adopted by ZigBee/802.15.4. By this mechanism, an ACK frame will be triggered by a successful reception of a DATA frame. The ACK frame must be sent back to its direct source node. As a result, the throughputs of the two parts in communication would be increased by the ACK mechanism. When the receiver sends an ACK frame, its out-throughput will increase; when the sender receives the ACK frame, its in-throughput will increase too.

2) Throughput Model of Uplink: After a ZigBee Mesh network has been constructed, the uplink (see Figure 3) is mainly used. And in the case of uplink communica- tion, the majority of frames yielded and being delivered are DATA and ACK frames. So, based on the Mesh topology model (see 3.1) and the ACK mechanism, the mathematical model for throughput in the case of uplink is proposed as follows.

Suppose that all the DATA frames yielded at node $i(i \in[2, n])$ are destined to node-1 (ZC), and all the frames can be transmitted and received successfully.

Definition 4: In uplinks, all the nodes that can yield frames and be routed by node $i$ are named as the uplink source nodes of node $i$ (denoted as $S u(i))$, then

$$
\operatorname{Su}(i)=\{d e(i), \operatorname{si}(i), \operatorname{de}(\operatorname{si}(i))\} \quad(i \in[2, n])
$$

The DATA frames yielded at $S u(i)$ would be routed by node $i$ with a probability $p_{u}\left(p_{u} \in(0,1]\right)$.

a) In-throughput of Uplink: First, all the input DATA frames of node $i$ during a time slice $T_{S}$ contribute to the uplink DATA in-throughput of node $i$ (denoted as $T P(i)_{u p-i n}^{\text {data }}$ ). Suppose the DATA frame yield rate at node $i$ is $\alpha(i)_{u}$. Here, $\alpha(i)_{u}$ takes the same value for any node $i \quad(i \in[2, n])$. Note that, because node- 1 is a $\mathrm{ZC}$ node, the DATA frame yield rate at node- 1 is $\alpha(1)_{u}=0$, but the ACK frame can still be yielded and emitted at node- 1 as other nodes. Then

$$
\begin{gathered}
T P(i)_{u p-i n}^{\text {data }}=\sum_{k \in s i(i)}\left(p(u)_{k i} \times \sum_{l \in d e(s i(k))}\left(\alpha(i)_{u} \times T_{S}\right)\right) \\
+\sum_{j \in d e(i)}\left(\alpha(i)_{u} \times T_{S}\right)(j, k, l \in S u(i))
\end{gathered}
$$

where the first item denotes the number of DATA frames that come from $s i(i)$; the second item denotes the number of DATA frames that come from $d e(i)$. Here, $\sum_{l \in \operatorname{de}(s i(k))}\left(\alpha(i)_{u} \times T_{S}\right)$ denotes the number of DATA frames that come from $\operatorname{de}(\operatorname{si}(i))$ to $\operatorname{si}(i)$.

Second, all the input ACK frames of node $i$ during $T_{S}$ contribute to the uplink ACK in-throughput of node $i$ (denoted as TP(i) $)_{u p-i n}^{a c k}$ ). Then

$$
T P(i)_{u p-i n}^{a c k}=T P(i)_{u p-i n}^{\text {data }}+\alpha(i)_{u} \times T_{S}
$$

where $\alpha(i)_{u} \times T_{S}$ denotes the number of ACK frames corresponding to the DATA frames yielded at node $i$.

Finally, all the input frames of node $i$ during $T_{S}$ contribute to the uplink total in-throughput of node $i$ (denoted as $\left.T P(i)_{u p-i n}^{\text {total }}\right)$. Then

$$
T P(i)_{u p-i n}^{\text {total }}=T P(i)_{u p-i n}^{\text {data }}+T P(i)_{u p-i n}^{a c k}
$$

b) Out-throughput of Uplink: First, all the output DATA frames of node $i$ during $T_{S}$ contribute to the uplink DATA out-throughput of node $i$ (denoted as $\left.T P(i)_{\text {up }- \text { out }}^{\text {data }}\right)$. Then

$$
T P(i)_{u p-o u t}^{\text {data }}=T P(i)_{u p-\text { in }}^{\text {data }}+\alpha(i)_{u} \times T_{S}
$$

where $\alpha(i)_{u} \times T_{S}$ denotes the number of DATA frames yielded at node $i$, which will be emitted. 
Second, all the output ACK frames of node $i$ during $T_{S}$ contribute to the uplink ACK out-throughput of node $i$ (denoted as TP(i) up-out $)$. Then

$$
T P(i)_{u p-o u t}^{a c k}=T P(i)_{u p-i n}^{d a t a}
$$

Finally, all the output frames of node $i$ during $T_{S}$ contribute to the uplink total in-throughput of node $i$ (denoted as $T P(i)_{u p-o u t}^{\text {total }}$ ). Then

$$
T P(i)_{u p-o u t}^{\text {total }}=T P(i)_{u p-o u t}^{\text {data }}+T P(i)_{u p-o u t}^{a c k}
$$

c) Total Throughput of Uplink: All the throughput (frames) of node $i$ during $T_{S}$ contribute to the uplink total throughput of node $i$ (denoted as $\left.T P(i)_{u p}^{\text {total }}\right)$. Then

$$
T P(i)_{u p}^{\text {total }}=T P(i)_{\text {up }- \text { in }}^{\text {total }}+T P(i)_{\text {up } \text { out }}^{\text {total }}
$$

According to Formulas (9)-(15), the uplink total throughput of node $i$ can be expressed as follows:

$$
\begin{gathered}
T P(i)_{u p}^{\text {total }}=4 \times T_{S} \times\left(2 \times \alpha(i)_{u}+\left(\sum_{j \in d e(i)}\left(\alpha(i)_{u}\right)\right.\right. \\
\left.\left.\quad+\sum_{k \in s i(i)}\left(p(u)_{k i} \times \sum_{l \in \text { de }(i(k)))}\left(\alpha(i)_{u}\right)\right)\right)\right)
\end{gathered}
$$

Finally, by modeling link selected probability and dynamic throughput of nodes etc., a comprehensive AGNM used for RERM is proposed.

\section{Simulation Experiments}

As mentioned above, the energy consumption is mainly determined by the throughput. The AGNM is designed for RERM. So its capability in characterizing dynamic throughput should be testified.

\subsection{Experiment Settings}

In the Cartesian coordinate plane $(\mathrm{x}, \mathrm{y})$, simulated nodes are $1(50,50), 2(75,0), 3(75,100), 4(100,50)$, $5(150,50), 6(175,0), 7(175,100), 8(100,150), 9(0$, 50 ), respectively. The ZRs (No.2-5) and ZEDs (No.6-9) sense environment information every 5 seconds. All the sensed information would be delivered to $\mathrm{ZC}$ (No.1).

The network topology adopted is a ZigBee Mesh topology (see Figure 1). The undirected link model $G_{t}=(V, E)$ can be expressed as an adjacency ma$\operatorname{trix} A_{G-t}$.

$$
A_{G-t}=\left[\begin{array}{ccccccccc}
0 & 1 & 1 & 1 & 0 & 0 & 0 & 0 & 1 \\
1 & 0 & 1 & 1 & 0 & 0 & 0 & 0 & 0 \\
1 & 1 & 0 & 1 & 0 & 0 & 0 & 1 & 0 \\
1 & 1 & 1 & 0 & 1 & 0 & 0 & 0 & 0 \\
0 & 0 & 0 & 1 & 0 & 1 & 1 & 0 & 0 \\
0 & 0 & 0 & 0 & 1 & 0 & 0 & 0 & 0 \\
0 & 0 & 0 & 0 & 1 & 0 & 0 & 0 & 0 \\
0 & 1 & 0 & 0 & 0 & 0 & 0 & 0 & 0 \\
1 & 0 & 0 & 0 & 0 & 0 & 0 & 0 & 0
\end{array}\right]
$$

The nodes relationship model $G_{r}=(V, E)$ (see Figures 2,3 ) can be expressed as adjacency matrix $A_{G-r}$.

$$
A_{G-r}=\left[\begin{array}{ccccccccc}
0 & 1 & 1 & 1 & 0 & 0 & 0 & 0 & 1 \\
0 & 0 & 1 & 1 & 0 & 0 & 0 & 0 & 0 \\
0 & 1 & 0 & 1 & 0 & 0 & 0 & 1 & 0 \\
0 & 1 & 1 & 0 & 1 & 0 & 0 & 0 & 0 \\
0 & 0 & 0 & 0 & 0 & 1 & 1 & 0 & 0 \\
0 & 0 & 0 & 0 & 0 & 0 & 0 & 0 & 0 \\
0 & 0 & 0 & 0 & 0 & 0 & 0 & 0 & 0 \\
0 & 0 & 0 & 0 & 0 & 0 & 0 & 0 & 0 \\
0 & 0 & 0 & 0 & 0 & 0 & 0 & 0 & 0
\end{array}\right]
$$

The uplink selected probability model $G(u)_{w e}=(V, E)$ (see Figure 4(b)) can be expressed as adjacency matrix $A_{U-1}$

$$
A_{U-l}=\left[\begin{array}{ccccccccc}
0 & 0 & 0 & 0 & 0 & 0 & 0 & 0 & 0 \\
0.80 & 0 & 0.10 & 0.10 & 0 & 0 & 0 & 0 & 0 \\
0.80 & 0.10 & 0 & 0.10 & 0 & 0 & 0 & 0 & 0 \\
0.60 & 0.20 & 0.20 & 0 & 0 & 0 & 0 & 0 & 0 \\
0 & 0 & 0 & 1.00 & 0 & 0 & 0 & 0 & 0 \\
0 & 0 & 0 & 0 & 1.00 & 0 & 0 & 0 & 0 \\
0 & 0 & 0 & 0 & 1.00 & 0 & 0 & 0 & 0 \\
0 & 0 & 1.00 & 0 & 0 & 0 & 0 & 0 & 0 \\
1.00 & 0 & 0 & 0 & 0 & 0 & 0 & 0 & 0
\end{array}\right]
$$

\subsection{Experimental Results}

For simplicity, we assumed that the electromagnetic environment was ideal and the throughput of each node was affordable, i.e. there was no delivery error.

1) Characteristics of Throughput: the characteristics of throughput in WSNs mainly include: dynamic throughput and dynamic components.

a) Dynamic Throughput: The AGNM can partially characterize the running state of WSNs, especially in terms of link selected probability and ACK mechanism. The dynamic throughput is an intuitive reflection of them.

Figure 5 shows the throughputs of nodes at time-slice (TS, $1 \mathrm{TS}=2$ hours) 1 and 12 . Comparing the cases of TS-1 with TS-12, the throughputs of node 1 (ZC) and node 6-9 (ZEDs) are static, and the throughputs of node 2-5 (ZRs) are dynamic. According to AGNM, the dynamic throughputs are caused by link selected probability (see Figure 4 (b)).

b) Dynamic Components: Because ACK mechanism is taken into account, the components of throughput have four types: DATA-out, ACK-out, ACK-in and DATA-in. 


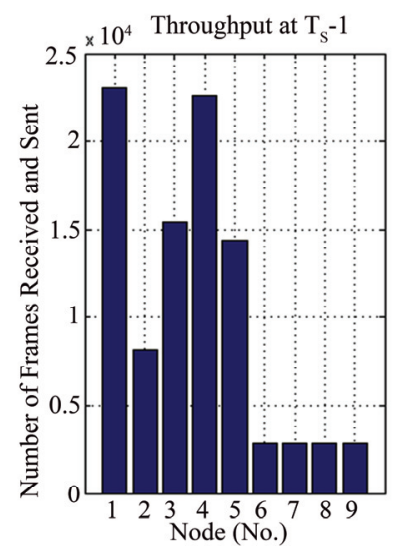

(a)

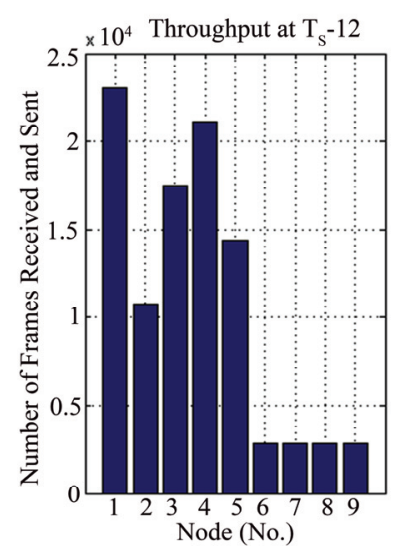

(b)
Figure 5. Throughputs of nodes. (a) at $\mathrm{T}_{\mathrm{S}} \mathbf{- 1}$; (b) at $\mathrm{T}_{\mathrm{S}}-12$.

Figure 6 shows the components of throughputs at TS-1 and TS-12. First, the components at node 1 (ZC) have no DATA-out and ACK-in, and the components at node 6-9 (ZEDs) have no DATA-in and ACK-out. After a PAN have been constructed, the cases shown in Figure 6 are normal in a ZigBee application (environment monitoring, etc.). Second, comparing the cases of TS-1 with TS-12, the components at node 1 (ZC) and node 6-9 (ZEDs) are static, and the components at node 2-5 (ZRs) are dynamic. Moreover, the changes of different components at node 2-5 (ZRs) from TS-1 to TS-12 are temporal processes (see Figure 7). According to the AGNM, the dynamic components were caused by link selected probability.

2) Performance Comparison: Compared with the network models (non-ACK supported) adopted by [7-10], the AGNM (ACK supported) have the advantage of characterizing the real situation of throughput (see Figures 8,9). So, the AGNM is a more application-oriented than those used in [7-10].

3) Analysis of Generality: The AGNM is a Meshsupported model. Because link selected probability is adopted, this model can be easily generalized to a Cluster-tree-supported model by setting some link selected probabilities to 0 in Figure 4(b), e.g. $\left\{\mathrm{p}_{2,1}=1, \mathrm{p}_{2,3}=0, \mathrm{p}_{2,4}\right.$ $=0, \mathrm{p}_{3,1}=1, \mathrm{p}_{3,2}=0, \mathrm{p}_{3,4}=0, \mathrm{p}_{4,1}=1, \mathrm{p}_{4,2}=0$ and $\left.\mathrm{p}_{4,3}=0\right\}$. So, the AGNM is more general than those used in [7-10].

4) Usage in RERM: The AGNM is mainly designed for RERM. So, it should be used in our RERM research first. The energy model adopted in this experiment was a simplified version of our previous work [6] (see Formula (20)).
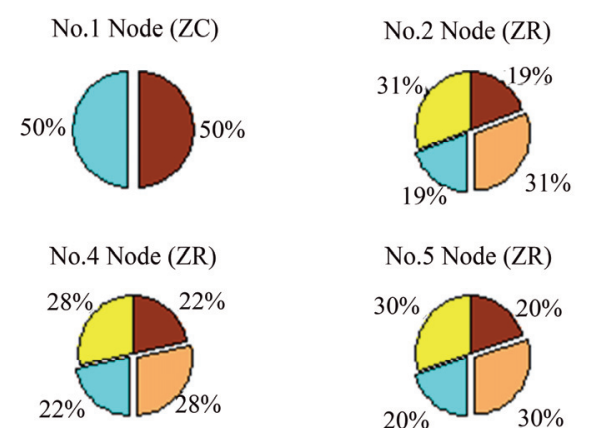

No.5 Node (ZR)
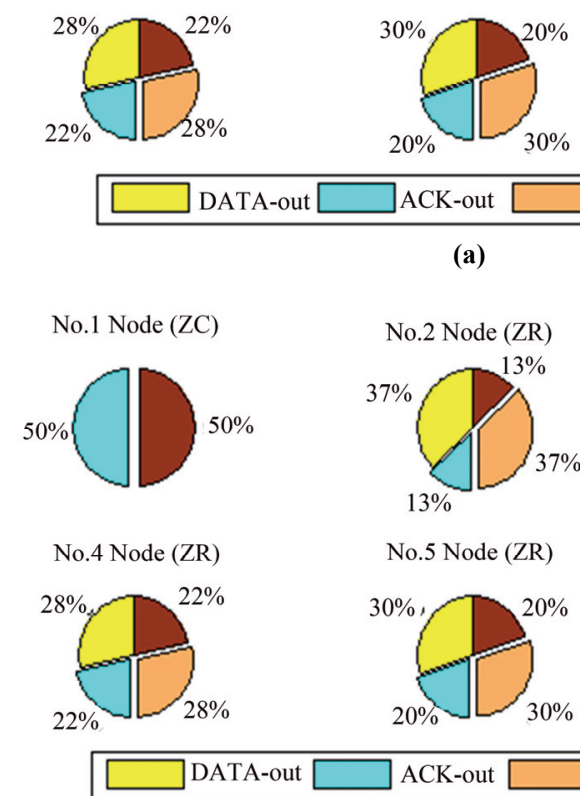

(a)

$$
\text { No. } 5 \text { Node (ZR) }
$$

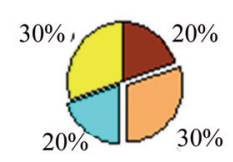

ACK-out $\square$ ACK-in

(b)
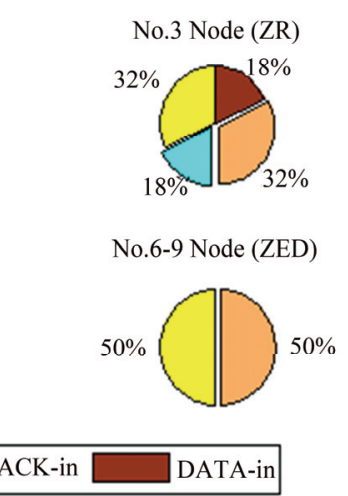

No.6-9 Node (ZED)

DATA-in

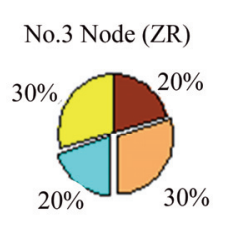

No.6-9 Node (ZED)

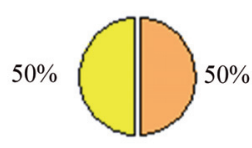

DATA-in

Figure 6. Components of throughput. (a) at TS-1; (b) at TS-12. 


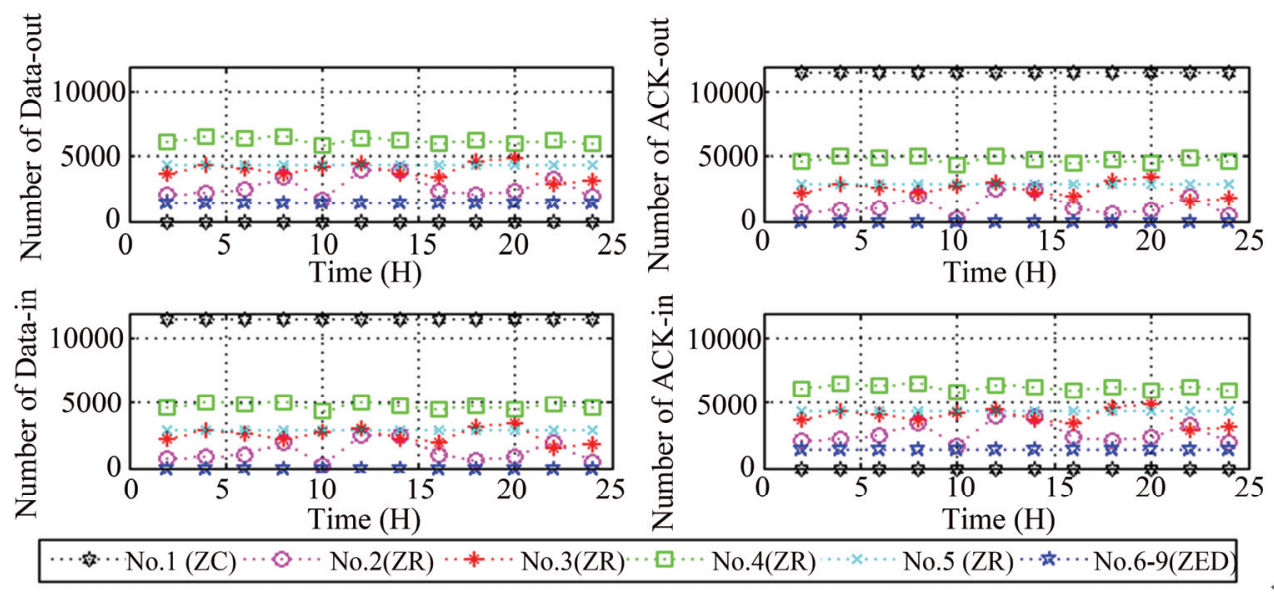

Figure 7. Components of throughput from TS-1 to TS-12.

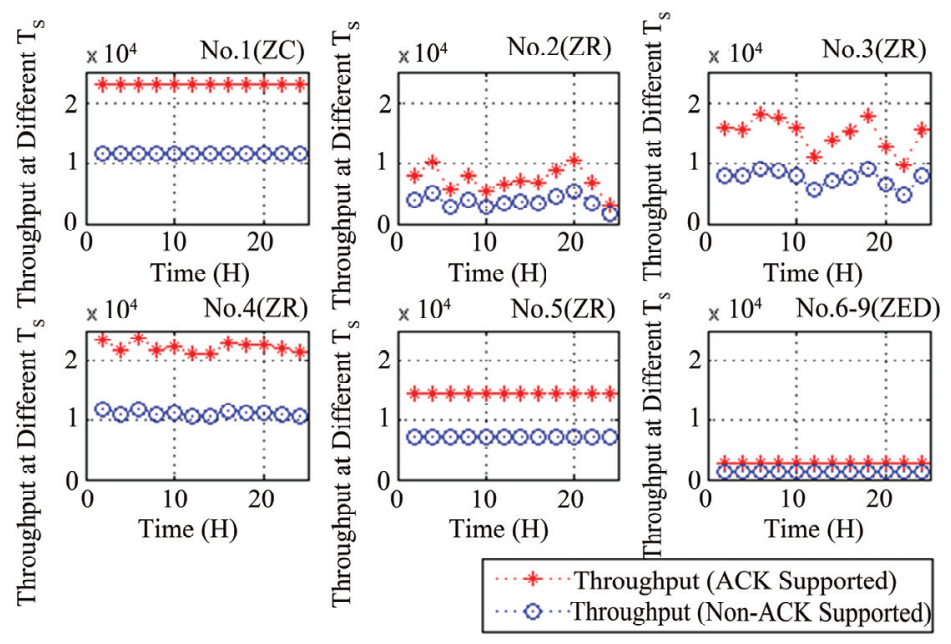

Figure 8. Throughput from TS-1 to TS-12.
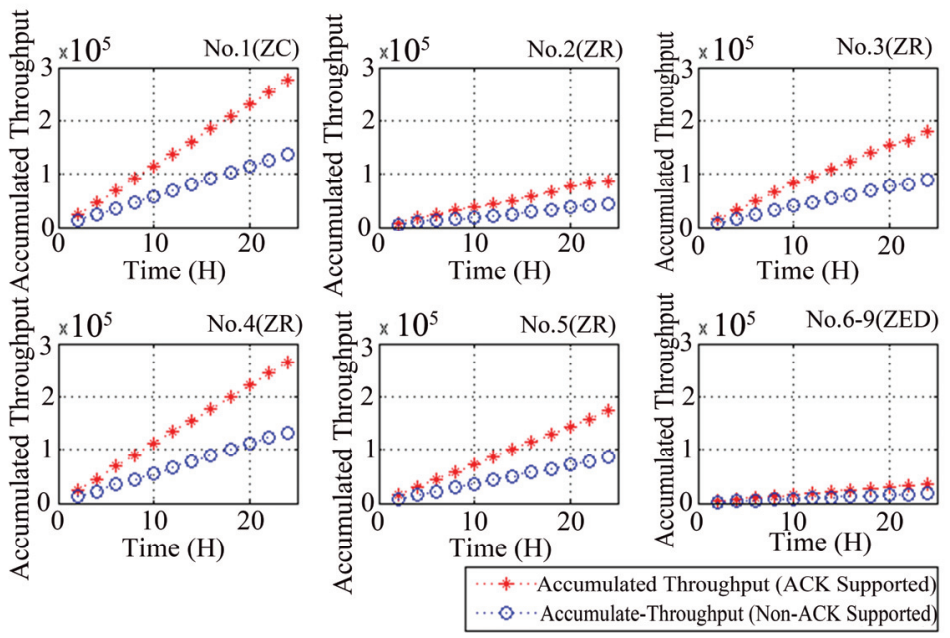

Figure 9. Accumulated throughput. 


$$
\begin{aligned}
E_{\text {dep }}= & \left(N_{\text {sen }} \times 266.571+N_{\text {rec }} \times 84.1158\right. \\
+ & 1.35 \times\left(7.2 \times 10^{6}-3.291 \times N_{\text {sen }}\right. \\
& \left.\left.-0.842 \times N_{\text {sen }}\right)\right) / 10^{3}(\mathrm{~mJ})
\end{aligned}
$$

Where $E_{\text {dep }}$ denotes the depleted energy, $N_{\text {sen }}$ denotes the out-throughput of a node, $N_{\text {rec }}$ denotes the in-throughput of a node. Besides, all the constants are computed based on MC13213 datasheet [17].

Suppose the initial energy reservation of a node (denoted as $\left.E_{\text {init }}\right)$ is $300 \mathrm{mAh}$, and let the residual energy of a node be $E_{\text {res }}$, then we have

$$
\begin{aligned}
E_{\text {res }}= & E_{\text {init }}-\left(N_{\text {sen }} \times 266.571+N_{\text {rec }} \times 84.1158\right. \\
& +1.35 \times\left(7.2 \times 10^{6}-3.291 \times N_{\text {sen }}\right. \\
& \left.\left.-0.842 \times N_{\text {sen }}\right)\right) / 10^{3}(\mathrm{~mJ})
\end{aligned}
$$

Based on the AGNM and Formula (20), the energy consumption of WSNs is simulated (see Figures 10,11).

Based on the AGNM and Formula (21), the residual energy of WSNs is simulated (see Figure 12) also.

Because node 1(ZC) has permanent power supply, its residual energy is always the same as $E_{\text {init }}$.

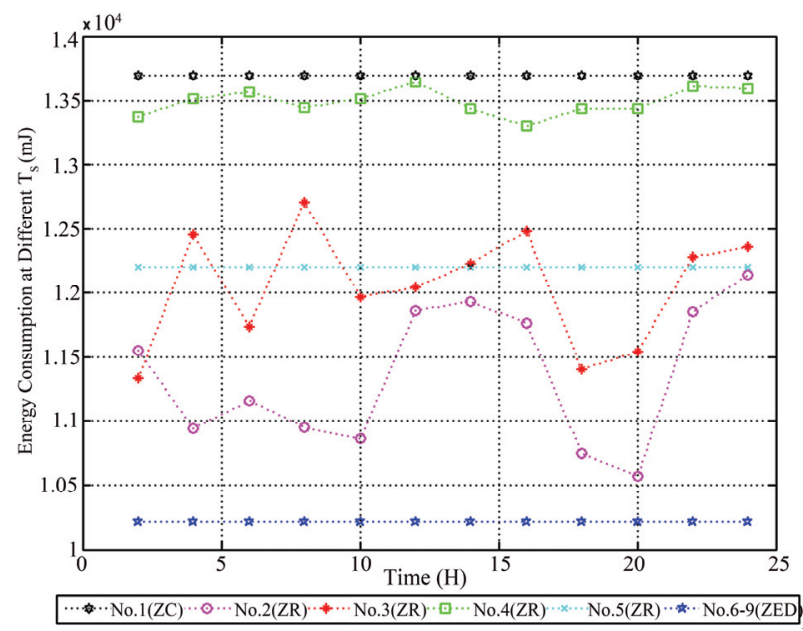

Figure 10. Energy consumption from TS-1 to TS-12.

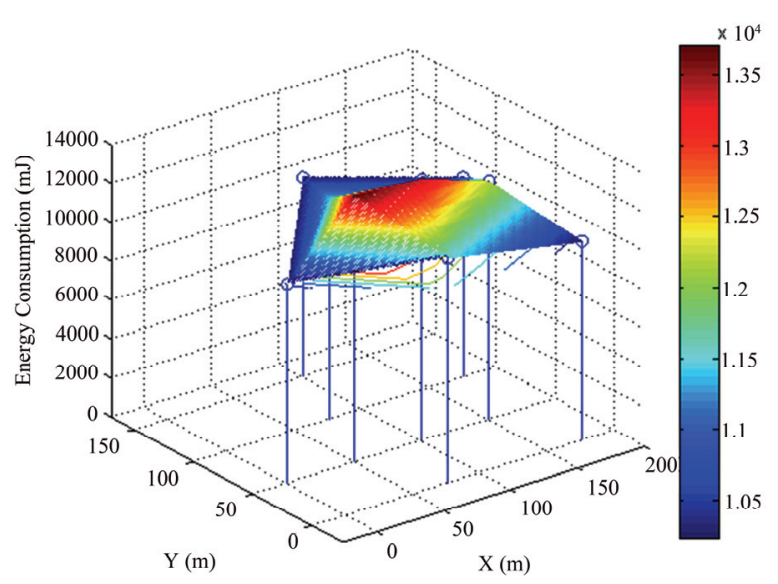

Figure 11. Contour of energy consumption at TS-1.

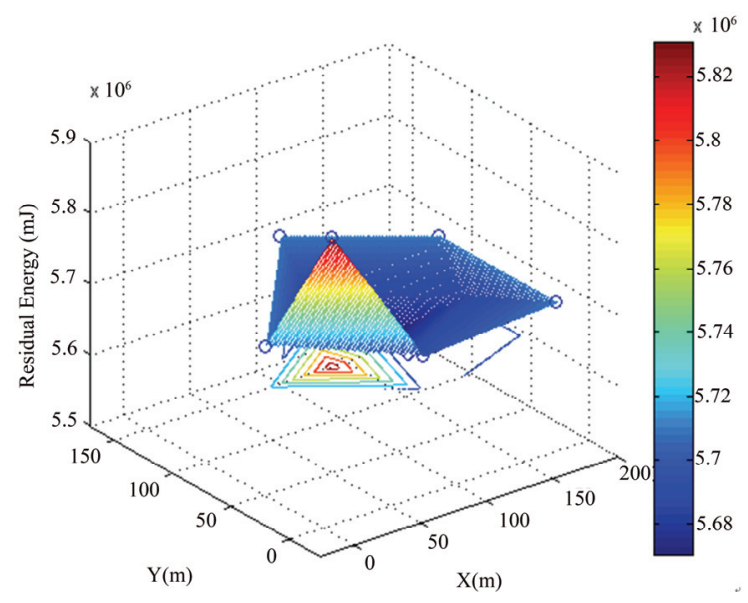

Figure 12. Contour of residual energy at TS-12.

In summary, because the AGNM can well characterize the link selected probability and ACK mechanism, the real situation of throughputs and energy consumption can be depicted in certain degree. Furthermore, the real situation of energy consumption can be partially characterized by the ANGM model.

\section{Conclusions and Future Works}

An application-oriented general network model (AGNM) designed for RERM research was proposed in this paper by encoding link selected probability and ACK mechanism etc. Different from the existing models, AGNM was built based on the practical Mesh topology, and it can be easily generalized to a Cluster-tree-supported one by setting some link selection probabilities to zero. The simulation results show that AGNM can approximately characterize some real properties of WSNs.

However, the throughputs of nodes are also influenced by other factors such as retransmission, which is introduced by heavy throughputs of neighbors and harsh electromagnetic environment etc. To better modeling the real situation of throughput, our future works will be focused on AGNM based retransmission inference and prediction algorithms.

\section{References}

[1] A. Mainwaring and D. Culler, "Wireless Sensor Networks for Habitat Monitoring," Proceedings of 1st ACM International Workshop on Wireless Sensor Networks and Applications, Atlanta, 2002, pp. 88-97.

[2] J. Paek and K. Chintalapudi, "Wireless Sensor Network for Structural Health Monitoring: Performance and Experience," Proceedings of 2nd IEEE Workshop on Embedded Networked Sensors, Sydney, 2005, pp. 1-10.

[3] A. Boukerche and H. A. B. Oliveira, "Localization Systems for Wireless Sensor Networks," IEEE Wireless Communi- 
cations, Vol. 14, No. 6, 2007, pp. 6-12.

[4] H. Edgar and J. Callaway, "Wireless Sensor Networks Architectures and Protocols," Auerbach Press, London, 2003.

[5] M. Z. Zamalloa and B. Krishnamachari, "An Analysis of Unreliability and Asymmetry in Low-Power Wireless Links," ACM Transactions on Sensor Network, Vol. 3, No. 2, 2007, p. 7.

[6] X. L. Cheng, Z. D. Deng and Z. R. Dong, "A Model of Energy Consumption Based on Characteristic Analysis of Wireless Communication and Computation," Journal of Computer Research and Development, Vol. 46, No. 12, 2009, pp. 1985-1993.

[7] Y. J. Zhao and R. Govindan, "Residual Energy Scans for Monitoring Wireless Sensor Networks," Proceedings. of IEEE Wireless Communication and Networking Conference, Marina Del Rey, Vol. 1, 2002, pp. 356-362.

[8] S. Han and E. Chan, "Continuous Residual Energy Monitoring in Wireless Sensor Networks," 2nd International Symposium on Parallel and Distributed Processing and Applications, Hong Kong, Vol. 3358, 2004, pp. 169-177.

[9] R. A. F. Mini, A. Loureiro and B. Nath, "PredictionBased Energy Map for Wireless Sensor Networks," Ad
Hoc Networks, Vol. 3, No. 2, 2005, pp. 235-253.

[10] X. Q. Meng and N. Thyaga, "Contour Maps: Monitoring and Diagnosis in Sensor Networks," Computer Networks, Vol. 50, No. 15, 2006, pp. 2820-2838.

[11] Software Technologies Group (STG), "How does ZigBee Compare with other Wireless Standards," 2006. http:// www.stg.com/wireless/ZigBee_comp.html

[12] Freescale Co., "Freescale BeeStack ${ }^{\mathrm{TM}}$ Software Reference Manual," 2008. http://www.freescale.com

[13] ZigBee Alliance, “ZigBee Document 053474r13”, 2006.

[14] IEEE Standard 802.15.4 TM $^{\mathrm{TM}}$ "Part 15.4: Wireless Medium Access Control and Physical Layer Specifications for Low-Rate Wireless Personal Area Networks", 2003.

[15] O. Kallenberg, "Foundations of Modern Probability," 2nd Edition, Springer, 2002.

[16] G. Ronald, "Graph theory," The Benjamin/Cummings Publishing Company, California, 1998

[17] Freescale Co., "MC132xx ZigBee ${ }^{\mathrm{TM}}$ - Compliant Platform - 2.4 GHz Low Power Transceiver for the IEEE® 802.15.4 Standard Plus Microcontroller," 2006. http://www.freescale.com 